\title{
Measurement in Sports Biomechanics
}

Simon Hood, MSc

S.Hood@tees.ac.uk (corresponding

author)

Thomas McBain

T.McBain@tees.ac.uk

Matt Portas, MSc

M.Portas@tees.ac.uk

Iain Spears, PhD

I.Spears@tees.ac.uk

Teesside University,

Middlesbrough

TS1 3BA, UK

\section{Abstract}

One of the major roles of a sports biomechanist or coach is to assess the movement patterns within sports performances. Movements can be analysed to enhance an individual's technique in terms of efficiency or to provide technical advantage. This paper aims to highlight the different measurement techniques available for the biomechanist to assess the movement characteristics of the technical and mechanical aspects of athletic performance.

\section{Introduction}

Incorporating elements of physics along with an understanding of functional movement and anatomy, the term biomechanics has been used for many years to describe the mechanical analysis of biological systems [1]. The study of biomechanics can therefore be applied to multiple disciplines and recently Bartlett expressed a definition of sports biomechanics as "the study and analysis of human movement patterns in sport" ( 11$)$ [2]. As coaches and performers strive for success the need to gain a competitive edge over an opponent is becoming increasingly important. Advances in technology over recent years have given developers opportunities to create systems to measure performance. With the advancements in academic and applied research, analysts are then able to use the systems to interpret the data produced and identify potential inefficiencies in an athlete's or team's performance. Based on the results of these analyses interventions can then be introduced and the effects on performance monitored using the same analysis systems. As technology has advanced the analyst can have confidence in the improved reliability of these test procedures. It is important to acknowledge that as scientists aiming to enhance performance, measurement techniques can range from live observations with immediate feedback to complex laboratory and fieldbased motion capture techniques, dependent on the movement being analysed, resources available and the type of feedback required. Coaches and analysts therefore need to be aware of the technological advancements that could lead to performance enhancement and it is the purpose of this review to consider the advances in measurement techniques in the field of sports biomechanics.

\section{Field Based Measurement Techniques}

Sports biomechanists have a range of techniques available to them and as an applied practitioner the methods used are determined by the movement to be analysed, the resources available and the situation in which performance is being measured. A common method of movement analysis comes in the form of technique analysis. Often based on live observation, coaches or biomechanists observe a performance, identify what they believe to be faults within the movement pattern and give feedback to the performer on how it can be improved, either through discussion or via a demonstration of the required movement [3]. This form of analysis, although readily available and costeffective, is not without its problems and inter-observer reliability (the degree to which different observers give consistent responses) of live observations has been reported with agreement between observers ranging from poor to good $[4,5]$.

In an attempt to enhance the reliability of observational data an alternative to live observation is the use of video analysis. Using a standard video camera movement patterns (training or competition based) can be recorded and reviewed, to provide feedback on the performance. Video analysis has become more accessible as technology has advanced with the introduction of higher specification cameras contained within mobile communication devices and tablet PCs. In a similar way to the live observations, with this method, ratings of inter-observer reliability have been reported from moderate to good [6]. However, the use of video enhances the feedback process with the availability of multiple play-backs of performances, slow motion playback, and the possibility of providing still images of significant points in the movement. On viewing the videos analysts are able to provide feedback in a timely manner to the performer in relation to whole body movement or about individual body segments, but, as with live observations the feedback is wholly subjective and based on the knowledge of the observer.

To provide objective feedback the analyst needs to turn to quantitative assessment tools to make judgements on a performance. Video analysis can be employed to provide useful objective feedback based on the quantification of events within a performance. For individual athletic events the effectiveness of this technique has been investigated in relation to sprint performance with elite $100 \mathrm{~m}$ sprinters [7]. In this research the authors used publicly available footage of elite 100m sprinters to quantify stride length and stride frequency characteristics displayed by individuals over a number of races. This provided insight for the first time into the world's fastest athletes in a competitive environment and highlighted the variations in individual performance patterns for these variables [7]. This shows how useful simple video analysis can be in progressing the biomechanist's understanding of movement and how it can provide ecologically valid data from a competitive arena which would not be replicable in a laboratory environment.

Live observation and video analysis are predominantly used to assess individual performances or provide subjective view points on a team's performance. The next technique outlined differs from observing a performance as it provides some quantification of movement characteristics, which is often required in team based sports. To assess teams or individuals within a team a technique known as notational analysis can be used. The notational analysis can be based on live observation or, more commonly, is completed using video analysis or semi-automated motion capture. The 
technique can be used to measure a number of different factors including physical, technical and tactical variables. The main aim is to track and monitor performance to compare inter (between different performers) or intra (the same person over different occasions) performance differences. A variety of systems are available and commercial systems mainly consist of motion capture or tracking systems to monitor performance over time. The notational analysis often considers factors that are required for a successful performance and these vary depending on the sport or activity being carried out [8]. The system requires a computer programme or note taker to monitor the performance counting the number of times something occurs (such as a completed pass in football). To develop a system the key factors of a successful and unsuccessful performance must be identified and this is where there is potential for inconsistencies in systems if the decision on what constitutes a successful performance is based on subjective criteria. For example, the system could be used to measure quantitative data on the number of occurrences of a specific action or distances covered, with some systems reportedly resulting in 95\% agreement between trained observers [9]. Further, the integration of motion capture or semiautomated tracking allows movement characteristics to be reported in relation to the direction of movement or the time spent moving at different velocities $[10,11]$.

In an attempt to improve performance, analysts and coaches aim to replicate the movement characteristics and physiological responses from competitive scenarios in training sessions. A limitation of semi-automated notational systems is that they rely on fixed camera locations often within stadiums therefore obtaining the same data in training is not possible as training often takes place away from competition venues. As movement data have not been readily available in training the intensity of sessions or training load have been measured in terms of physiological responses using heart rate monitors. The lack of movement data from training poses a problem for analysts and coaches as they attempt to replicate match scenarios.

Alternative approaches to notational analysis have been reported and Global Positioning System (GPS) technology has advanced the way in which competitive and training environments can be monitored. The use of GPS technology with integrated accelerometers allows a performer's movement to be tracked. To do so the athlete wears a small unit, normally between the shoulders, weighing approximately $0.3 \mathrm{Kg}$ with a sampling frequency between 1 and $10 \mathrm{~Hz}$. The GPS technology allows the generation of real time movement data and immediate feedback on whole body movement. At present some sport's governing body regulations such as the Federation of International Football Association's (FIFA) prohibit the wearing of such devices during competitive matches [11]. However, the wearing of such devices is allowed in other football codes such as rugby league and it is also seen in practice during non-competitive soccer fixtures. Research has been conducted into the validity of GPS systems in relation to generic movement data (linear and multidirectional) $[12,13]$ and there has also been an attempt to compare GPS data in relation to semi-automated tracking devices during non-competitive performances $[14,15]$. As the current literature demonstrates agreement between devices, training in some sports can now be planned based on the data from notational analysis and semi-automated tracking and then monitored using GPS (where resources allow).

In summary, the field based techniques outlined can be used to provide subjective feedback on performance or objective measures of whole body movement of individuals or teams. Often issues with performance are highlighted using the field based measures that require a more detailed investigation. This may be through further quantitative analysis of the whole body or by measuring specific limb or body segments that are active within a movement.

\section{Laboratory Based Measurement Techniques}

Biomechanics is often used to assess movement to enhance an individual's performance. To gain a deeper understanding of movements laboratory based techniques can be implemented to obtain information on the kinetics (forces in relation to time) and kinematics (movement in relation to time) of the movement.

\subsection{Force platform/inserts}

The measurement of forces is essential to gain an understanding of human movement and a common laboratory based technique is the use of a force platform. A force platform incorporates a number of force transducers built into a solid plate. When an object or limb makes contact with the plate the force of the movement is applied to the plate and a reaction force is then applied back to the object. The built in force transducers measure the force and it can be displayed in terms of three performance planes (vertical, anteriorposterior and medio-lateral). This enables them to be used to measure ground reaction forces during a plethora of gait and jumping related sporting activities.

Force plates can sample at up to $2000 \mathrm{~Hz}$ and numerous kinetic variables can be observed in relation to performance. A problem with the use of force plates to assess athletic performance is that they are often fixed within the laboratory setting and this can limit the movements that can be analysed and could lead the performer to adapt their usual movement pattern to contact the force plate. For example, it has been reported that when a performer 'targets' a force plate they might make movement adaptations dependent on the stride length used [16]. Thus caution should be taken when measuring some variables when a performer is specifically asked to contact a force plate during movement. Portable force plates are now available which can be used outside of the laboratory environment, which allows for movements to be carried out in a more ecologically valid environment but the problem of targeting still remains.

\subsection{Pressure plates}

Although multiple variables can be determined from a force plate output biomechanists are often interested in the distribution of force over a given surface area. This is particularly relevant when conducting assessments of running or walking patterns. To measure the magnitude of force over a given area a pressure plate could be used. Pressure plates are available in different sizes and allow barefoot and shod measurements in static and dynamic conditions (using appropriate gait software). The pressure plate provides additional information on temporal and spatial factors and many biomechanists focus on how the foot rolls on contacting the plate during gait [17]. The centre of pressure throughout the foot contact can be determined and data on the spread 

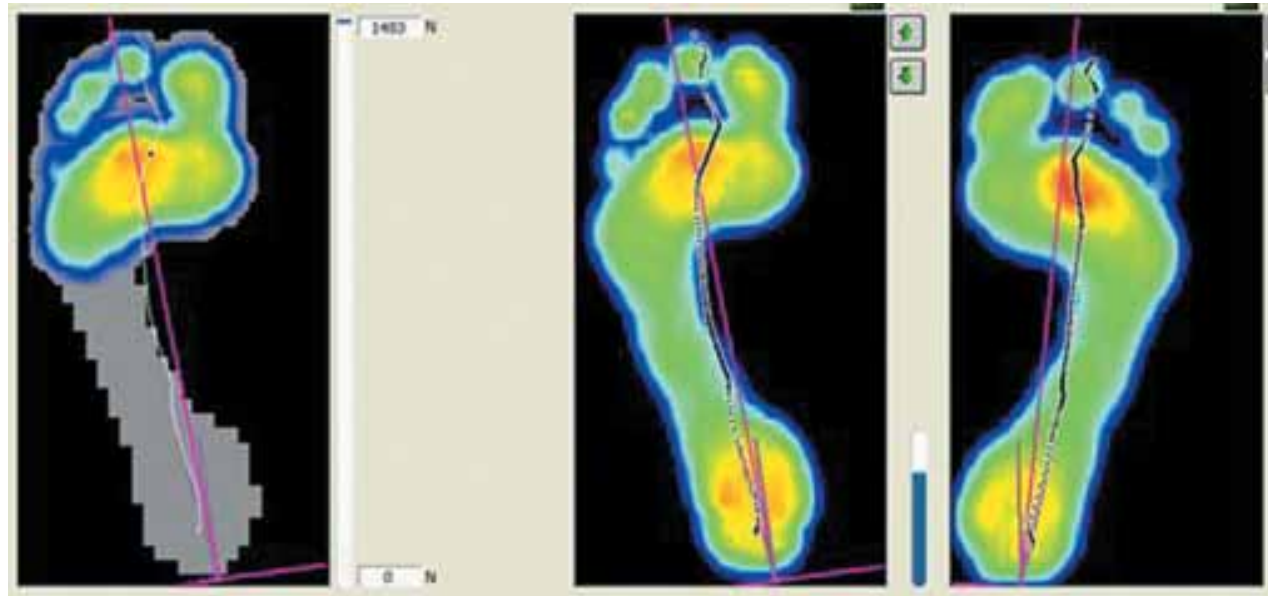

\subsection{Manual and automated digitising}

In addition to the measurement of forces it is of interest to have an understanding of the movement patterns generated by the body or segments within a whole body movement. Kinematic analysis refers to the study of movement in relation to space and time and considers such variables as displacement, velocity and acceleration. Actions may be analysed in two or three dimensions depending on the complexity of the movement.

Two-dimensional analysis (also referred to as 2-D kinematics) is often used to measure movements in a single performance plane or when

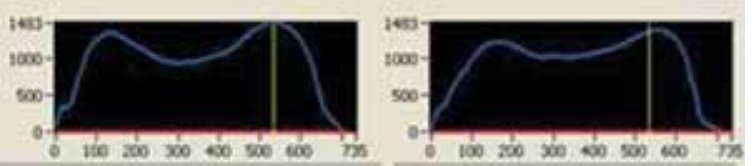
sophisticated equipment is not available as in the main it relies on the use of a single video camera or recording device. The camera should be positioned in such a way to produce the clearest possible image of the action and guidelines for filming have been

Figure 1: Visual pressure analysis output during barefoot walking (data were collected using a RSfootscan $0.5 \mathrm{~m}$ plate, Gait 7.0 Second Generation, RSScan International, Belgium)

of force across different segments of the foot can be obtained (as illustrated in Figure 1). The darker areas refer to higher levels of pressure throughout the foot contact. The image on the left highlights that the performer does not roll fully onto the first meta-tarsal region during the toe-off phase of gait with most pressure being exerted in the region of the second and third meta-tarsal during the toe-off stage of walking. The data produced can be used to identify potential causes of injury and the data presented in Figure 1 suggest that the participant's foot is in a slightly inverted position at toe-off. This may be due to a high arch in the foot which could result in the participant being susceptible to ankle and heel pain, lateral knee pain or problems with the lower back, during periods of prolonged activity. presented [2]. The speed of the movement often presents problems as standard video cameras do not have a sufficient sampling frequency to capture the full detail of high speed movements. An example would be trying to capture a golf swing using a standard camera, the top of the backswing and start of the follow through may be captured but not include ball contact, thus high speed cameras are often preferred to standard cameras to allow an increased frame rate during capture.

Digitising the movement requires the use of computer software to map the coordinates of limb segments and joint centre locations during the movement (see Figure 2). Using basic mathematical principles such as trigonometry and an appropriate calibration of the capture space, linear and angular data can be produced

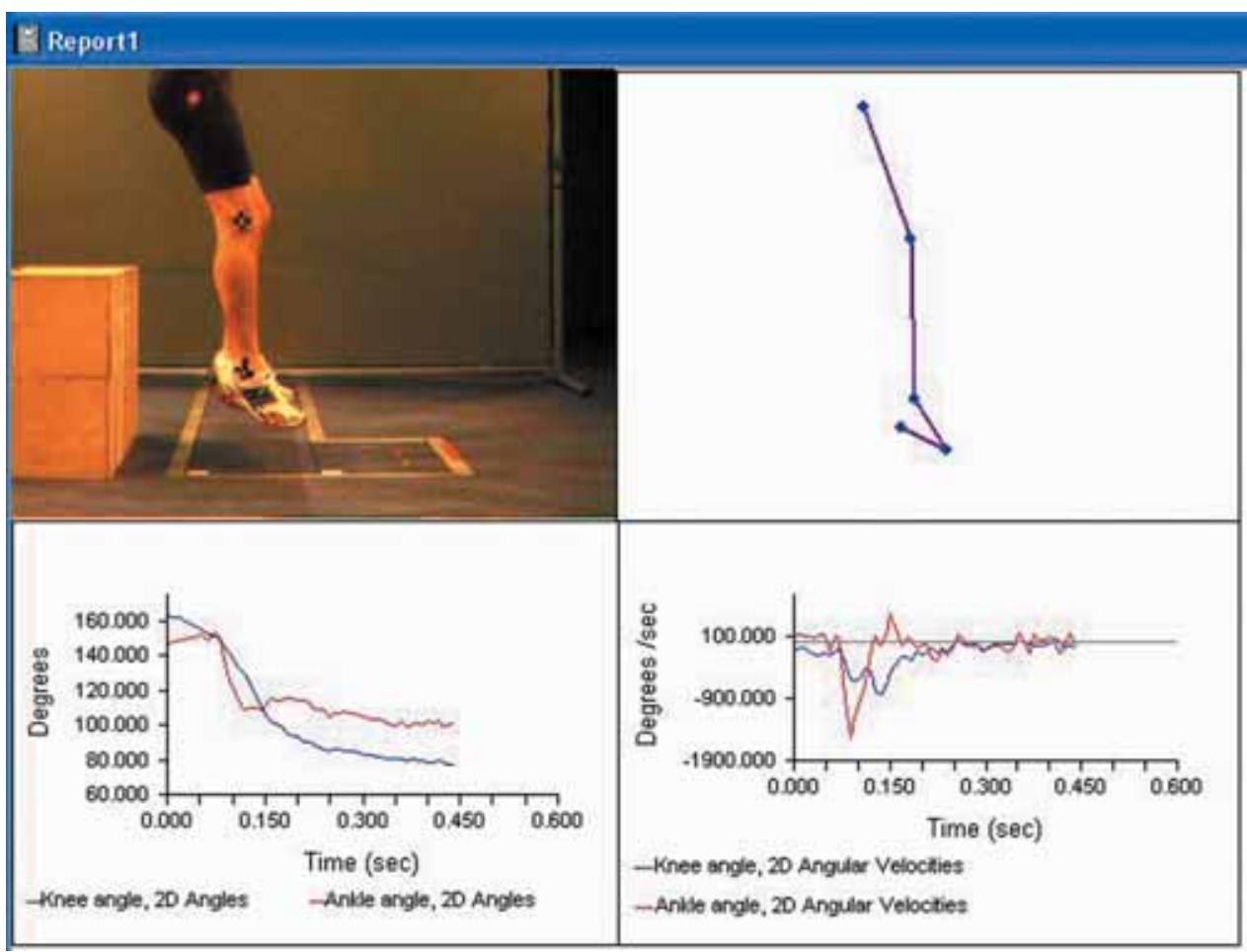

Figure 2: Sample screen shot of 2-D kinematic analysis using Vicon Motus version 9 (Vicon Motion Systems, Oxford) for the movement. This is not without its problems as the data produced can be affected by perspective and parallax error and therefore the accuracy of the camera set up is essential to ensure consistency in data collection.

To advance the kinematic analysis and consider movements that cross multiple planes (as in most sporting movements) three-dimensional (3-D) analysis is required. 3-D analysis can be performed with computer software linked to multiple video cameras; however, optical motion capture techniques are now more widely used which incorporate the use of infra-red cameras and reflective markers placed on the athlete (as seen in Figure 3). Similar to 2-D analysis 3-D kinematics requires the determination of joint centre locations and subsequent reconstruction of the joint during movements through expressing the joint-centre location in relation to a local reference frame or marker set on a particular segment [18]. Previous clinical approaches to joint centre location have used invasive methods requiring pins to specifically locate joint centres. Although 
evidently more valid, these are not applicable to dynamic assessment in sporting movements, and a more widely used non-invasive method for both clinical and sport environments is the application of reflective markers [19] Motion capture systems record the location of the markers on the bony landmarks and the geometry of the bones and joint axes are determined using standardised algorithms based on vector algebra and allow for the deduction of joint kinematics and kinetics [20]. As we might expect, the method is not without flaws. The underlying geometry is assumed upon the location of surface lying markers for every subject and does not account for individual differences. Also the marker placement, that is, the location of landmarks is a repeatable operation and open to human error with poor marker placement impacting on the subsequent kinetic and kinematic variables produced [20].

Although high validity and reliability values for 3-D analysis have been reported for gait analysis and it is referred to as the gold standard method [21], caution must be exerted when attempting to measure sporting performances due to the laboratory based nature of the technique and the lack of ecological validity in some testing protocols.

A rapidly expanding alternative approach to measuring human movement is the use of Inertial Measurement Systems or Units (IMSs or IMUs). These are becoming common in biomechanics research [22, 23] and in performance assessment [24]. IMUs are small electronic devices that usually contain gyroscopes, magnetometers and accelerometers that can be fixed onto an object or onto an athlete's limb segments. The data from each of the sensors can be processed to determine the 3-D orientation of the segment onto which the IMU is mounted. Specifically, gyroscopes measure the rate of turn about 3 axes which can be integrated with respect to time to deliver a rotation matrix. The accelerometers and magnetometers are used to de-drift the orientation matrix in the vertical and horizontal planes, respectively. These calculations can be done almost instantaneously even on low-cost micro-processors, and, using wireless data transmission real-time data can be sent to a receiving device. When multiple segments from adjacent limbs are transmitted, the orientation matrices can be linked together to form 3-dimensional real-time displays of human movement.

A selection of laboratory based techniques for measuring kinematic and kinetic data have been outlined and can be advantageous when interpreted by the biomechanist to give a greater understanding of a movement. As stated the laboratory based nature of the techniques can often limit the movements that can be analysed. To advance the analysis 3-D motion capture provides data in this environment which can be used to validate the IMUs. If the IMUs are then validated for a particular movement they can subsequently be used to collect data in the field, to provide a more ecologically valid analysis.

\section{Research in Practice}

Biomechanical analysis involves a large amount of motion tracking, usually concentrating on performance parameters including force and speed, however, the methods of capturing these variables differs depending upon the setting. The benefits of 2-D and 3-D motion capture are unrivalled for capturing dynamic skills such as a golf swing and sprint starts in a laboratory setting but are sometimes not feasible in an applied setting such as out on the golf course or in an athletics stadium. The identification of low-cost, practical yet accurate instruments is therefore of great interest to biomechanists.

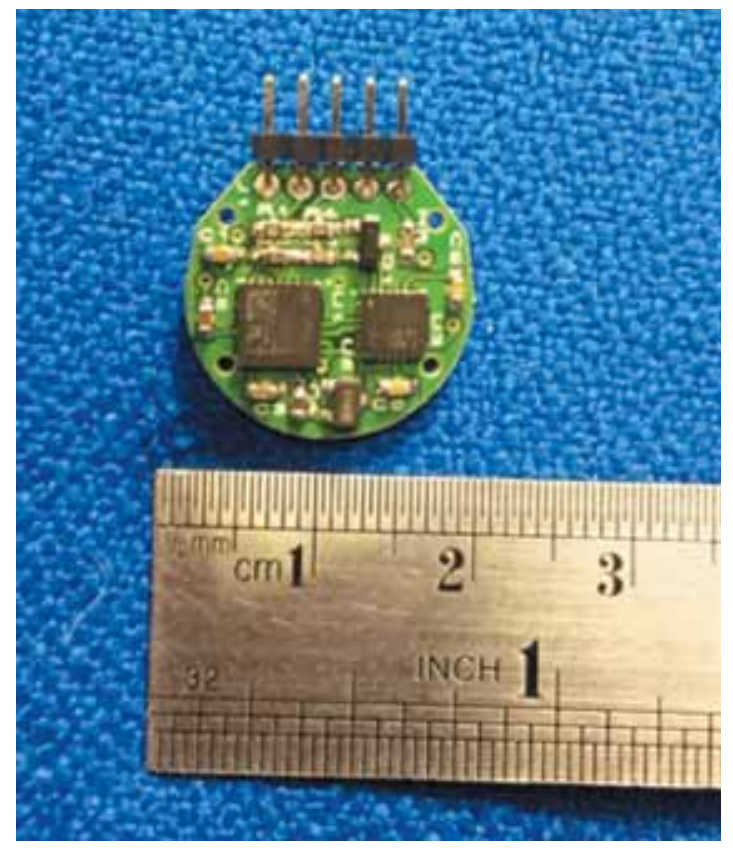

Figure 4: Custom made Inertial Measurement Unit (IMU) 
In our laboratories at Teesside University we have developed and tested IMUs that can provide reliable data (unpublished data) similar to that obtained through expensive cinematography (motion capture). Algorithms designed for the IMUs enable the calculation of specific performance measures where data can be accessed instantly to inform practice and improve training. The units are roughly the size of a 1 pence piece (see Figure 4) and transmit wirelessly to a computer. They can be attached to individual body segments or to sports peripherals to measure velocity, acceleration and rotations. We have primarily used the units in boxing research measuring peak acceleration of the hand during punches landed on a punch bag, impact force upon the bag and number of punches. The rotational outputs also allow for the tracking of particular movements meaning they are capable of distinguishing between jabs, hooks and uppercuts in boxing. They offer direct practical application of real-time performance measures. In addition to technique analysis, the devices can be used to assess effects of training interventions. For example, we are currently using IMUs to assess the effects of nutritional supplements on training performance in elite boxers, in terms of punch frequency and punch impulse.

\section{Conclusions}

The aim of this paper was to introduce a selection of measurement techniques that are used in the assessment of movement in sport. Although an account is presented of the main techniques employed by sports biomechanists, it should be noted that the account is not exhaustive. Other techniques such as electromyography and isokinetic dynamometry are also commonly used to quantify sport performance. These techniques range from simple observations of movement to the use of advanced technology but the most vital aspect is the skill of the biomechanist in interpreting the data and feeding back to the athlete.

Examples have been provided to demonstrate how measurement in sports biomechanics can underpin subsequent performance enhancement. As stated the type of measurements taken are determined by the movement being analysed, resources available and the situation in which performance is being measured. It is also important to note that although advances in technology will enhance the capacity to measure biomechanical aspects of performance, potential suggestions for interventions arising from these observations must not be made in isolation. An interdisciplinary approach is required which also includes physiological and psychological expertise together with knowledge from the coaching team. In this way, the biomechanical analysis can be expected to deliver real improvements in sports performance.

\section{References}

[1] S. J. Hall, Basic Biomechanics, 6th ed. New York: McGraw-Hill, 2012.

[2] R. Bartlett, Introduction to Sports Biomechanics, Analysing Human Movement Patterns, 2nd ed. New York: Routledge, Taylor and Francis, 2009.

[3] A. Lees, "Technique analysis in sports: a critical review", Journal of Sport Sciences, vol. 20, pp. 813-828, Mar. 2002 [4] T. A. L. Wren, S. A. Rethlefsen, B. S. Healy, K. Patrick, A. W. Dennis and R. M. Kay. "Reliability and validity of visual assessments of gait using a modified physician rating scale for crouch and foot contact", Journal of Pediatric Orthopaedics, vol. 25, pp. 646-650, 2005.

[5] S. Barris and C. Button, "Review of vision-based motion analysis in sport", Sports Medicine, vol. 38, pp. 1025-1043, 2008.
[6] J. J. Brunnekreff, C. J. T. van Uden, S. van Moorsel and J. G. M. Kooloos, "Reliability of videotaped observational gait analysis in patients with orthopedic impairments", BMC Musculoskeletal Disorders, vol. 6(17), pp. 1-9, Mar. 2005.

[7] A. I. T Salo, I. N. Bezodis, A. M. Batterham and D. G. Kerwin "Elite sprinting: Are athletes individually step-frequency or step-length reliant?", Medicine and Science in Sports and Exercise, vol. 43, pp. 1055-1062, Jun. 2011.

[8] M. Hughes and I. Franks, " Notational analysis of sport." London: E. \& F.N. Spon, 1997.

[9] P. Bradley, P. O'Donoghue, B. Wooster and P. Tordoff, "The reliability of ProZone MatchViewer: a video-based technical performance analysis system", International Journal of Performance Analysis in Sport, vol. 7, pp. 117-129, Oct. 2007. [10] V. Di Salvo, W. Gregson, G. Atkinson, P. Tordoff and B. Drust, "Analysis of high intensity activity in Premier League Soccer", International Journal of Sports Medicine, vol. 30, pp. 205-212, 2009.

[11] R. M. L. Barros, M. S. Misuta, R. P. Menezes, P. J. Figueroa, F. A. Moura, S. A. Cunha, R. Anido and N. J. Leite, "Analysis of the distances covered by first division Brazilian soccer players obtained with an automatic tracking method", Journal of Sports Science and Medicine, vol. 6, pp. 233-242, Mar. 2007 [12] H. Macleod, J. Morris, A. Nevill and C. Sunderland, "The validity of a non-differential global positions system for assessing player movement patterns in field hockey", Journal of Sports Sciences, vol. 27, pp. 121-128, Jan. 2009.

[13] M. D. Portas, J. A. Harley, C. A. Barnes and C. J. Rush, "The validity and reliability of $1-\mathrm{Hz}$ and $5-\mathrm{Hz}$ global positioning systems for linear, multidirectional and soccer specific activities", International Journal of Sports Physiology and Performance, vol. 5, pp. 448-458, Dec. 2010.

[14] J. A. Harley, C. A. Barnes, M. D. Portas, R. Lovell, S. Barrett, D. Paul and M. Weston, "Motion analysis of match-play in elite U12 to U16 age-group soccer players", Journal of Sports Sciences, vol. 28, pp. 1391-1397, Nov. 2010.

[15] J. A. Harley, R. J. Lovell, C. A. Barnes, M. D. Portas and M. Weston, "The interchangeability of GPS and semiautomated video-based performance data during elite soccer match play", Journal of Strength and Conditioning Research, vol. 25, pp. 2334-2336, 2011

[16] J. H. Challis, "The variability in running gait caused by force plate targeting", Journal of Applied Biomechanics, vol. 17, pp. 77-83, 2001.

[17] T. M. Willems, D. De Clerq, K. Delbaere, G. Vanderstraeten, A. De Cock and E. Witvrouw, "A prospective study of gait related risk factors for exercise-related lower leg pain", Gait and Posture, vol. 23, pp. 91-98, Jan. 2006.

[18] A. Roosen, M. T. G. Pain and M. Begon, "Limitations of functionally determined joint centres for the analysis of athletic human movement: a case study of the upper limb", Journal of Applied Biomechanics, vol. 25, pp. 281-292, 2009.

[19] R. M. Ehrig, W. R. Taylor, G. N. Duda and M. O. Heller, "A survey of formal methods for determining the centre of rotation of ball joints", Journal of Biomechanics, vol. 39, pp. 2798-2809, 2006

[20] I. W. Charlton, P. Tate, P. Smyth and L. Roren, "Repeatability of an optimised lower body model", Gait and Posture, vol. 20, pp. 213-221, Oct. 2004.

[21] A. H. Mackey, S. E. Walt, G. A. Lobb and S. N. Stott, "Reliability of upper and lower limb three-dimensional kinematics in children with hemiplegia", Gait and Posture, vol. 22, pp. 1-9, Aug. 2005.

[22] H. Tan, A. M. Wilson and J. Lowe, "Measurement of stride parameters using a wearable GPS and inertial measurement unit", Journal of Biomechanics, vol. 41, pp. 1398-1406, Feb. 2008.

[23] A. Kruger and J. Edelmann-Nusser, "Biomechanical analysis in freestyle snowboarding: application of a full-body inertial measurement system and a bilateral insole measurement system", Sports Technology, vol. 2, pp. 17-23, 2009.

[24] E. Bergamini, P. Picerno, H. Pillet, F. Natta, P. Thoreux and V. Camomilla, "Estimation of temporal parameters during sprint running using a trunk-mounted inertial measurement unit." Journal of Biomechanics, vol. 45, pp. 1123-1126, Apr. 2012. 